\title{
Coronavirus disease 2019 (COVID-19) and antibiotic stewardship: Using a systems engineering approach to maintain patient safety
}

\author{
Julie A. Keating $\mathrm{PhD}^{1}$, Linda McKinley $\mathrm{MPH}^{1}$ and Nasia Safdar $\mathrm{MD}, \mathrm{PhD}^{1,2}$ \\ ${ }^{1}$ William S. Middleton Memorial Veterans' Hospital, Madison, Wisconsin and ${ }^{2}$ Department of Medicine, University of Wisconsin-Madison School of Medicine and \\ Public Health, Madison, Wisconsin
}

To the Editor-In the absence of effective treatments for coronavirus disease 2019 (COVID-19), many hospitalized COVID-19 patients receive antibiotics. ${ }^{1,2}$ Thus far, the literature does not indicate that antibiotics are effective in treating COVID-19, and the incidence of bacterial coinfections appears low. ${ }^{2}$ One analysis reported that while $8 \%$ of COVID-19 patients experienced a bacterial or fungal coinfection, $72 \%$ of COVID-19 patients received antibiotics. $^{2}$

Clostridioides difficile infection (CDI) is associated with broad-spectrum antibiotics frequently used for COVID-19; CDI is thus a significant concern for COVID-19 patients. ${ }^{3}$ Patients at higher risk of severe COVID-19 frequently also have risk factors for CDI such as advanced age and weakened immune systems. ${ }^{3}$ COVID-19 treatments themselves, which often involve extended hospital stays, can also increase a patient's risk of developing healthcare-associated $\mathrm{CDI}^{3}{ }^{3} \mathrm{CDI}$ has been identified in patients who received antibiotics as part of their COVID-19 treatment. ${ }^{4,5}$

Given the patient safety risks posed by CDI, effective antibiotic stewardship remains critical throughout the COVID-19 pandemic. However, pandemic-related changes to healthcare delivery (eg, drug shortages, changing pharmacy workflows, and redeployed healthcare workers) have made antibiotic stewardship interventions even more challenging. We present a systems engineering approach to evaluate and modify antibiotic stewardship programs within the constraints of COVID-19 responses, with the overall goal of reducing CDI.

\section{Systems Engineering Initiative for Patient Safety (SEIPS): A framework to support antibiotic stewardship}

Antibiotic stewardship initiatives can involve persuasive (eg, education or audit with feedback) and restrictive (eg, formulary restriction) approaches; these initiatives are used together with appropriate diagnostic and infection prevention measures. These strategies require complex behavioral changes and can involve significant resources such as real-time access to antibiotic stewardship staff for consultation.

The COVID-19 pandemic response has further increased the complexity of antibiotic stewardship. ${ }^{6}$ Prescribers and antibiotic stewardship team members are facing higher and more complex patient loads. The wide-ranging symptoms of COVID-19 may mimic other infections, and a worsening of symptoms is frequently

\footnotetext{
Author for correspondence: Nasia Safdar, E-mail: ns2@medicine.wisc.edu

Cite this article: Keating JA, McKinley L, and Safdar N. (2021). Coronavirus disease 2019 (COVID-19) and antibiotic stewardship: Using a systems engineering approach to maintain patient safety. Infection Control \& Hospital Epidemiology, 42: 1416-1418, https://doi.org/10.1017/ice.2020.1263
}

seen 1-2 weeks into the disease that can make it difficult to identify potential coinfections. The length of hospitalization for many patients increases risks of developing healthcare-acquired infections such as ventilator-associated pneumonia that may require additional antibiotic treatment. ${ }^{7}$ These factors, combined with a lack of effective treatment options for severe COVID-19, have resulted in high levels of antibiotic use among COVID-19 inpatients. $^{1,2}$

The structure and effectiveness of an antibiotic stewardship program is dependent on the individual work-system context, including characteristics of the patient population, organizational culture toward antibiotic stewardship, availability of infectious disease and pharmacy personnel, accessibility of clinical decision support tools, and existing policies to support antibiotic stewardship. A systems engineering approach can be used (1) to fully evaluate the roles that work-system elements play in complex antibiotic stewardship interventions and (2) to develop modifications to these elements to support the implementation of interventions. The Systems Engineering Initiative for Patient Safety (SEIPS) provides a framework for this approach. SEIPS defines work-system elements: person(s), technology and tools, environment, tasks, and organization. The interaction of these elements influences care processes and outcomes. 8

Given the urgent needs to ensure appropriate antibiotic use and reduce CDI risk in COVID-19 patients, a systems engineering approach such as SEIPS can be used to understand the various work-system factors that are involved in antibiotic stewardship and CDI prevention. The interaction of these elements drives antibiotic stewardship, COVID-19 treatment, and CDI prevention processes in each work system; thus, they influence critical patient and organizational outcomes. This SEIPS-based approach provides flexibility for teams to evaluate their own work-system-specific barriers and facilitators to antibiotic stewardship practices within their COVID-19 response. ${ }^{9}$ Teams can then develop strategies to support antibiotic stewardship within the individual work system to optimize patient and organizational outcomes.

\section{Work system-based elements can be modified to support antibiotic stewardship throughout COVID-19 response}

Table 1 lists strategies based on SEIPS work-system elements to support antibiotic stewardship and CDI prevention through COVID-19 responses. The SEIPS framework is Person centered: the COVID-19 patient, prescribers, and antibiotic stewardship team members interact with all work-system elements. People use Technology and Tools (eg, antibiotic prescription decision

(c) The Author(s), 2020. Published by Cambridge University Press on behalf of The Society for Healthcare Epidemiology of America. This is an Open Access article, distributed under the terms of the Creative Commons Attribution licence (http://creativecommons.org/licenses/by/4.0/), which permits unrestricted re-use, distribution, and reproduction in any medium, provided the original work is properly cited. 
Table 1. Practices to Support Antibiotic Stewardship and Reduce CDI in the Care of COVID-19 Patients

\begin{tabular}{|c|c|}
\hline SEIPS Element & Antibiotic Stewardship Practice \\
\hline \multirow{3}{*}{ Person(s) } & Avoid antibiotic use unless patient is severely ill or in case of bacterial coinfection \\
\hline & Prioritize infectious diseases and/or pharmacy availability for antibiotic prescription consultations \\
\hline & Provide education for staff regarding antibiotic stewardship and COVID-19 treatments \\
\hline \multirow{4}{*}{$\begin{array}{l}\text { Technology and } \\
\text { tools }\end{array}$} & Develop facility guidelines for antibiotic prescribing to COVID-19 patients \\
\hline & Build in review periods for updates and evidence-based practices for antibiotic use during COVID-19 treatments \\
\hline & Build antibiotic prescribing decision support tools into the electronic medical record for COVID-19 patients \\
\hline & $\begin{array}{l}\text { Ensure that virtual communication tools are available as part of workflow for infectious diseases and pharmacy consultations } \\
\text { regarding antibiotic prescribing }\end{array}$ \\
\hline \multirow[t]{7}{*}{ Organization } & Involve antibiotic stewardship team in COVID-19 response and incident command ${ }^{10}$ \\
\hline & Consider joint antibiotic stewardship-infection preventionist team to develop facility's COVID-19 antibiotic stewardship response \\
\hline & Regularly review antibiotic use and outcomes in COVID-19 patients and revise facility antibiotic stewardship guidelines accordingly \\
\hline & Maintain existing antibiotic stewardship programs and practices for patients with and without COVID-19 \\
\hline & Communicate regularly regarding facility guidelines for antibiotic use in COVID-19 patients \\
\hline & Ensure leadership support and commitment to continued antibiotic stewardship and CDI prevention initiatives \\
\hline & Include CDI risk as part of rounds for COVID-19 patients \\
\hline \multirow[t]{7}{*}{ Tasks } & Continue to track and report overall antibiotic use and resistance rates as well as antibiotic use specifically in COVID-19 patients \\
\hline & Track and report CDI rates overall and specifically in COVID-19 patients \\
\hline & Follow diagnostic and testing guidelines for bacterial and fungal coinfections in COVID-19 patients prior to initiating antibiotic therapy \\
\hline & Use existing antibiotic stewardship guidelines to treat secondary infections in COVID-19 patients (eg, urinary tract infection) \\
\hline & $\begin{array}{l}\text { Monitor and postpone antibiotic use in stable COVID-19 patients with potential healthcare-acquired infections (eg, ventilator-associated } \\
\text { pneumonia) }\end{array}$ \\
\hline & Review COVID-19 patient medical history for prior CDI and CDI risk factors when determining treatment plans \\
\hline & Review and update antibiotic stewardship workflows to account for COVID-19 response-related changes (eg, redeployed personnel) \\
\hline \multirow[t]{4}{*}{ Environment } & Review facility's pre-existing CDI rates and use rates of CDI-associated antibiotics (eg, fluoroquinolones) \\
\hline & $\begin{array}{l}\text { Monitor antibiotic availability due to pandemic-related shortages and develop alternative therapy plans to avoid use of broad- } \\
\text { spectrum antibiotics where possible }\end{array}$ \\
\hline & Add visual reminders (eg, signs) about antibiotic use in COVID-19 patients and the need to continue existing CDI prevention practices \\
\hline & $\begin{array}{l}\text { Ensure infection control practices for CDI remain a priority, such as hand hygiene with soap and water, environmental cleaning, and } \\
\text { appropriate diagnostic testing }\end{array}$ \\
\hline
\end{tabular}

Note. CDI, Clostridioides difficile infection.

support tools built into electronic medical records) to enact Tasks (eg, choosing appropriate therapies for the patient, including postponing or de-escalating antibiotics). The Organization's antibiotic stewardship and infection control infrastructure should continue activities such as reporting antibiotic usage, resistance, and CDI rates. Within the work-system Environment, visual cues reminding prescribers of best practices for antibiotic prescribing in COVID-19 patients may be useful given the high and complex patient loads. Existing CDI prevention practices (eg, environmental cleaning and appropriate diagnostic testing) should remain a priority.

Research is needed to understand whether, when, and how antibiotics should be used to treat COVID-19 patients while minimizing adverse effects such as CDI. CDI in COVID-19 patients should be investigated to identify risk factors such as use of specific antibiotics, previous CDI, and/or presentation of COVID-19 gastrointestinal symptoms. Research findings should be incorporated into comprehensive evidence-based antibiotic stewardship programs. In the meantime, the urgency of preventing CDI in COVID-19 patients requires adjusting antibiotic stewardship interventions to fit within current COVID-19 protocols. The SEIPS-based approach presented here can help local antibiotic stewardship teams and decision makers adjust existing plans and develop new approaches to support antibiotic stewardship and reduce CDI during the COVID-19 pandemic.

Acknowledgments. The views expressed in this article are those of the authors and do not necessarily reflect the position or policy of the Department of Veterans' Affairs or the United States government.

Financial support. This material is based upon work supported by the Veterans' Health Administration, Quality Enhancement Research Initiative (grant no. HX003040).

Conflicts of interest. The authors report no potential conflicts of interest related to this article. 


\section{References}

1. Zhou F, Yu T, Du R, et al. Clinical course and risk factors for mortality of adult inpatients with COVID-19 in Wuhan, China: a retrospective cohort study. Lancet 2020;395:1054-1062.

2. Rawson TM, Moore LSP, Zhu N, et al. Bacterial and fungal coinfection in individuals with coronavirus: a rapid review to support COVID-19 antimicrobial prescribing. Clin Infect Dis 2020;ciaa530. doi: 10.1093/cid/ciaa530.

3. Spigaglia P. COVID-19 and Clostridioides difficile infection (CDI): possible implications for elderly patients. Anaerobe 2020;64:102233.

4. Sandhu A, Tillotson G, Polistico J, et al. Clostridioides difficile in COVID-19 patients, Detroit, Michigan, USA, March-April 2020. Emerg Infect Dis 2020;26:2272-2274.

5. Páramo-Zunzunegui J, Ortega-Fernández I, Calvo-Espino P, et al. Severe Clostridium difficile colitis as potential late complication associated with COVID-19. Ann R Coll Surg Engl 2020:e1-e4.
6. Huttner BD, Catho G, Pano-Pardo JR, Pulcini C, Schouten J. COVID-19: don't neglect antimicrobial stewardship principles! Clin Microbiol Infect 2020;26:808-810.

7. François B, Laterre PF, Luyt CE, Chastre J. The challenge of ventilatorassociated pneumonia diagnosis in COVID-19 patients. Crit Care 2020;24:289.

8. Holden RJ, Carayon P, Gurses AP, et al. SEIPS 2.0: a human factors framework for studying and improving the work of healthcare professionals and patients. Ergonomics 2013;56:1669-1686.

9. Getahun H, Smith I, Trivedi K, Paulin S, Balhy H. Tackling antimicrobial resistance in the COVID-19 pandemic. Bull WHO 2020; 98:442-442A.

10. Stevens MP, Patel PK, Nori P. Involving antimicrobial stewardship programs in COVID-19 response efforts: all hands on deck. Infect Control Hosp Epidemiol 2020;41:744-745.

\title{
Risk factors associated with carbapenem-resistant Klebsiella pneumoniae bloodstream infections in a tertiary-care hospital in India
}

\author{
Priyanka Gupta $\mathrm{DNB}^{1}$ (1), Neha Bollam BS², Yatin Mehta FRCA ${ }^{3}$, Sharmila Sengupta MD ${ }^{1, a}$ and Sumanth Gandra MD ${ }^{4, a}$ \\ ${ }^{1}$ Department of Microbiology, Medanta, The Medicity Hospital, Gurugram, Haryana, India, ${ }^{2}$ University of North Carolina, Chapel Hill, North Carolina, \\ United States, ${ }^{3}$ Institute of Critical Care and Anaesthesiology, Medanta, The Medicity Hospital, Gurugram, Haryana, India and ${ }^{4}$ Department of Internal Medicine, \\ Division of Infectious Diseases, Washington University, School of Medicine, St. Louis, Missouri, United States
}

To the Editor-Klebsiella pneumoniae is the second most frequently isolated organism from blood cultures after Escherichia coli in India, with $50 \%$ of all $K$. pneumoniae isolates being resistant to meropenem in $2019 .{ }^{1}$ Infections due to carbapenem-resistant K. pneumoniae (CRKP) are difficult to treat and have serious implications for patient health and medical costs. ${ }^{2}$ Two recent meta-analyses ${ }^{3,4}$ reported the risk factors of CRKP infections; however, none of the studies included in the meta-analyses were from India or South Asia, where CRKP infections are highly prevalent. ${ }^{5}$ The high burden of CRKP in Indian healthcare settings necessitates investigation into the risk factors for CRKP infections to identify potential interventions. Here, we examined the risk factors associated with CRKP bloodstream infections (BSIs) compared to carbapenem-sensitive K. pneumoniae (CSKP) BSIs in the Indian context.

A retrospective study was conducted at Medanta-The Medicity, a tertiary-care hospital with 1,500 beds in North India between August 2014 and July 2015. Patients with BSI caused by K. pneumoniae were included in the study. Only the first BSI with K. pneumoniae from a patient was included. Data were collected by reviewing medical records. The variables included demographics, admission diagnosis, ICU admission, comorbidities, exposure to invasive devices, immune status, prior exposure to antibiotics,

Author for correspondence: Priyanka Gupta, E-mail: priyankavkgupta@gmail.com ${ }^{a}$ Authors of equal contribution.

Cite this article: Gupta P, et al. (2021). Risk factors associated with carbapenemresistant Klebsiella pneumoniae bloodstream infections in a tertiary-care hospital in India. Infection Control \& Hospital Epidemiology, 42: 1418-1420, https://doi.org/ $10.1017 /$ ice.2020.1280 length of hospital stay and outcome. The blood-culture specimens were processed using BacT/Alert 3D (bioMérieux, Marcy l'Etoile, France) blood-culture system. Identification and antibiotic susceptibility testing was performed using a Vitek 2 compact instrument (bioMérieux, Marcy l'Etoile, France). The Clinical and Laboratory Standards Institute (CLSI 2015) break points were utilized. ${ }^{6}$ Categorical variables were reported as frequencies and percentages, and continuous variables were reported as medians and interquartile ranges. For univariate analyses, results were reported as odds ratios with $95 \%$ confidence intervals and $\mathrm{P}$ values. Variables with $\mathrm{P}$ values $<.10$ in the univariate analyses were included in backward, stepwise, logistic regression to determine the final multivariate logistic regression model to evaluate risk factors for CRKP. Statistical analyses were performed using SPSS Statistics 26 software (IBM, Armonk, NY). The study protocol was reviewed and approved by the institutional review board of Medanta Hospital, and the requirement for obtaining informed consent was waived because of the study's retrospective design.

Overall, during the study period, 111 patients had K. pneumoniae BSI. Of 111,85 patients (77\%) had CRKP and 26 patients $(23 \%)$ had CSKP BSI. In univariate analysis, the following variables were associated with CRKP BSI: prior use of carbapenems, prior ICU stay, presence of foley catheter, mechanical ventilation, admission to ICU, prior use of polymyxins, admission to gastroenterology service and admission to liver transplant service (Table 1). However, in multivariate analysis, prior carbapenem use (odds ratio [OR], 7.04; 95\% confidence interval [CI], $1.86-26.68 ; P=.004)$, presence of Foley catheter (OR-6.21; 95\% CI- 1.61-23.98; $P=.008)$ and admission to gastroenterology service

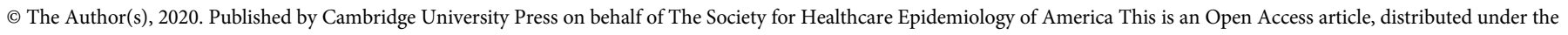

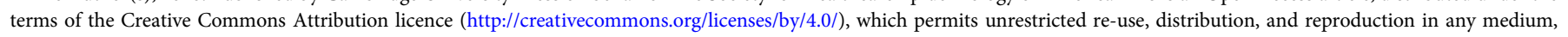
provided the original work is properly cited. 